\title{
Mortality risk of triglyceride levels in patients with coronary artery disease
}

\author{
Takatoshi Kasai, ${ }^{1}$ Katsumi Miyauchi, ${ }^{1}$ Naotake Yanagisawa, ${ }^{1}$ Kan Kajimoto, ${ }^{2}$ \\ Naozumi Kubota, ${ }^{1}$ Manabu Ogita, ${ }^{1}$ Shuta Tsuboi, ${ }^{1}$ Atsushi Amano, ${ }^{2}$ Hiroyuki Daida ${ }^{1}$
}

'Departments of Cardiology, Juntendo University, School of Medicine, Tokyo, Japan ${ }^{2}$ Departments of Cardiovascular Surgery, Juntendo University, School of Medicine, Tokyo, Japan

\section{Correspondence to}

Dr Hiroyuki Daida, Department of Cardiology, Juntendo University, School of Medicine, 2-1-1 Hongo, Bunkyo-ku, Tokyo 113-8421, Japan; daida@juntendo.ac.jp

Received 3 July 2012 Revised 21 August 2012 Accepted 21 August 2012 Published Online First 26 September 2012

\section{SLinked}

- http://dx.doi.org/10.1136/ heartjnl-2012-302968

To cite: Kasai T, Miyauchi K, Yanagisawa N, et al. Heart 2013, 99, 22-29.

\section{ABSTRACT}

Objective The association between triglyceride level and the risk of coronary artery disease (CAD) remains controversial. In particular, the prognostic significance of triglyceride levels in established CAD is unclear. We aimed to assess the relationship between triglyceride levels and long-term (>10 years) prognosis in a cohort of patients after complete coronary revascularisation.

Design Observational cohort study.

Setting Departments of cardiology and cardiovascular surgery in a university hospital.

Patients Consecutive patients who had undergone complete revascularisation between 1984 and 1992. All patients were categorised according to the quintiles of fasting triglyceride levels at baseline.

Main outcome measures The risk of fasting triglyceride levels for all-cause and cardiac mortality was assessed by multivariable Cox proportional hazards regression analyses.

Results Data from 1836 eligible patients were assessed. There were 412 (22.4\%) all-cause deaths and 131 (7.2\%) cardiac deaths during a median follow-up of 10.5 years. Multivariable analyses including total and high-density lipoprotein cholesterol and other covariates revealed no significant differences in linear trends for allcause mortality according to the quintiles of triglyceride ( $p$ for trend $=0.711$ ). However, the HR increased with the triglyceride levels in a significant and dose-dependent manner for cardiac mortality ( $p$ for trend=0.031). Multivariable analysis therefore showed a significant relationship between triglyceride levels, when treated as a natural logarithm-transformed continuous variable, and increased cardiac mortality (HR 1.51, $\mathrm{p}=0.044$ ).

Conclusions Elevated fasting triglyceride level is associated with increased risk of cardiac death after complete coronary revascularisation.

Several epidemiological studies have investigated the relationships between serum triglyceride levels and morbidity and mortality rates of coronary artery disease (CAD) ${ }^{1-4}$ However, the evidence for elevated triglyceride levels as an independent risk factor for CAD remains controversial because there is no uniformity in data obtained in large epidemiological studies. There is a concern that adjustment for other abnormal lipid profiles, such as high-density lipoprotein (HDL) cholesterol levels, attenuates the relationship between triglycerides and CAD because there is an inverse correlation between triglyceride levels and HDL cholesterol levels. Nevertheless, meta-analyses have shown that serum triglyceride levels are an independent risk factor for morbidity and mortality rates of CAD in primary prevention. ${ }^{5-8}$

Unlike primary prevention, there are few data on the long-term prognostic significance of triglyceride levels in secondary prevention of CAD. The relationship between triglyceride levels and mortality risk after complete coronary revascularisation has not been established. We aimed to assess the relationship between triglyceride levels and long-term prognosis in a cohort of patients with CAD after complete coronary revascularisation.

\section{METHODS \\ Subjects}

We analysed data from consecutive patients who had undergone coronary revascularisation, including percutaneous coronary intervention (PCI) and coronary artery bypass grafting (CABG), at Juntendo University Hospital (Tokyo, Japan) between January 1984 and December 1992. We included patients who had achieved complete revascularisation-that is, patients in whom no unbypassed major vessels had a stenosis of $\geq 50 \% .{ }^{9}{ }^{10}$ Patients with an untreated neoplasm at baseline and those with associated complex cardiac procedures such as valve replacement or aneurysm repair at the time of surgical revascularisation were excluded. The study was approved by the institute's internal review board and was performed according to the principles expressed in the Declaration of Helsinki and the ethics policy of the institute.

\section{Data collection and definitions of variables}

Demographic data including age, gender, body mass index (BMI), coronary risk factors, medication use, revascularisation procedure-related factors and comorbidities were prospectively collected. Blood samples were obtained in the early morning after an overnight fast. Hypertension was defined as systolic blood pressure $\geq 140 \mathrm{~mm} \mathrm{Hg}$ and diastolic blood pressure $\geq 90 \mathrm{~mm} \mathrm{Hg}$ or treatment with antihypertensive medications. Diabetes mellitus (DM) was defined as fasting plasma glucose level of $\geq 6.99 \mathrm{mmol} / \mathrm{l}$ or treatment with oral hypoglycaemic drugs or insulin injections. A current smoker was defined as a patient who smoked at the time of complete revascularisation or who had quit smoking within 1 year prior to the procedure. Patients with isolated PCI had achieved complete revascularisation by PCI without bypass grafting. 


\section{Outcomes}

The follow-up period ended on 30 September 2000. Survival data were collected by establishing serial contact with the patients or their families or from the medical records of deceased patients or those who continued to be followed up at our hospital. Information about the circumstances and date of death was obtained from the families of patients in cases where the patient died at home, and details of the events or the cause of death was supplied by other hospitals or clinics where the patients were admitted. Mortality data were categorised according to the causes of death (eg, all-cause or cardiac deaths) using the International Classification of Diseases, Ninth Revision codes 410-414, 785.51 and 798 .

\section{Statistical analysis}

For the main analysis, all patients were categorised according to the quintiles of triglyceride levels. Continuous variables are expressed as mean \pm SD and categorical data are tabulated as frequencies and ratios. Differences between the baseline characteristics of patients within each triglyceride category were analysed by analysis of variance for continuous variables and the Cochrane-Armitage test for trend for proportions. To determine whether the results differed with the cut-off points, we performed secondary analyses in which triglyceride levels were treated as a natural logarithm-transformed continuous variable.

Cumulative mortalities were plotted using Kaplan-Meier curves and differences between quintiles of triglyceride levels were determined using log-rank tests. $p$ Values for log-rank trend tests were also estimated. Cox proportional hazard models were used to compute HR and 95\% CI for each quintile of triglyceride level using the lowest quintile as the reference group. Linear trend analyses were performed by using linear contrast coefficients $(-2,-1,0,1,2)$ in Cox proportional hazard models. The assumption of proportional hazards was assessed using a log-minus-log survival graph. Models were initially adjusted for age and gender (Model 1). To determine the role of triglycerides independent of other lipid markers, we adjusted the models for total and HDL cholesterol levels (Model 2). Furthermore, multivariable models were adjusted for non-HDL and HDL cholesterol levels (Model 3) and for BMI, presence of hypertension, presence of DM, current smoking, family history of $\mathrm{CAD}$, prior myocardial infarction (MI), prior stroke, presence of atrial fibrillation, under dialysis, left ventricular ejection fraction, number of diseased vessels, presence of an arterial bypass to left anterior descending artery, presence of a left main trunk lesion, whether complete revascularisation was achieved by isolated PCI and use of drugs (aspirin, angiotensin-converting enzyme (ACE) inhibitors, $\beta$-blockers, statins, fibrates and niacin) in addition to the variables used in Model 2 (Model 4). To avoid overadjustment, the latter covariates were added only if they were significant predictors of death from all-cause or cardiac death at an $\alpha$ level of 0.1 . Finally, multivariable models were further adjusted for non-HDL cholesterol levels plus the same variables as in Model 4 other than total cholesterol (Model 5).

To assess the potential heterogeneity of the effect of triglyceride levels on cardiac mortality we performed subgroup analyses. The subgroups included age groups (cut-off 60 years), gender, presence/absence of DM, total cholesterol (cut-off $5.69 \mathrm{mmol} / \mathrm{l}$ ), HDL cholesterol (cut-off $1.29 \mathrm{mmol} / \mathrm{l}$ ) and use of statins. The first-order interactions in multivariable Cox proportional hazards models were examined by entering interaction terms between triglyceride levels and the abovementioned subgroup variables. We also determined the effect of triglyceride levels on cardiac mortality in each subgroup.

A $p$ value of $<0.05$ was considered statistically significant unless indicated otherwise. All data were analysed using SAS V.9.2 (SAS Institute, Cary, North Carolina, USA).

\section{RESULTS}

We assessed data from 1836 eligible patients who had undergone complete coronary revascularisation during the study period. Baseline and clinical event data were fully documented during a median follow-up period of 10.5 years. All patients underwent PCI with simple balloon angioplasty; no patient received stent implantation since stents were not available when complete revascularisation was achieved. All CABG procedures were performed using a conventional cardiopulmonary bypass; arterial grafts were used in $51.4 \%$ of cases. None of the patients had type 1 DM. During the follow-up period 412 patients $(22.4 \%)$ died from any cause and 131 patients $(7.2 \%)$ died from cardiac causes.

The baseline characteristics of patients by quintiles of triglyceride levels are shown in table 1 . Patients with high triglyceride levels were likely to be young, male and current smokers with a high BMI and total cholesterol level, a low HDL cholesterol level and frequently had prior MI. Among patients with high triglyceride levels, a smaller percentage of patients underwent revascularisation by isolated PCI, a high percentage were taking aspirin and a low percentage were taking statins.

The cumulative survival curves of patients according to the quintiles of triglyceride levels are shown in figure 1. Patients with high triglyceride levels were more likely to have high cumulative cardiac mortality rates (figure $1 \mathrm{~B}$ ) but they did not show any trend to high cumulative all-cause mortality (figure 1A).

The results of Cox proportional hazard regression analyses for all-cause and cardiac mortality are summarised in figure 2 . Linear trends for all-cause mortality according to the quintiles of triglyceride levels were not significant in any models except for Model 1. However, among each quintile of triglyceride level in all models, HR increased significantly with the triglyceride levels in a dose-dependent manner for cardiac mortality.

The results of Cox proportional hazard regression analyses, in which triglyceride levels were treated as natural logarithmtransformed continuous variables, are also shown in figure 2 . For all-cause mortality, only Model 1 showed a significant association between logarithm-transformed triglyceride level and mortality. However, for cardiac mortality, all models showed significant associations between these two factors.

We also conducted a subgroup analysis separately from the age, gender, presence of DM, total and HDL cholesterol levels and the use of statins for all-cause and cardiac death. Although associations of triglyceride level with mortality were more prominent in men, patients with low HDL and patients not receiving statins, all $\mathrm{p}$ values for interaction were not significant (figure 3).

\section{DISCUSSION}

In this study we made several important findings that provide insights into the relationship between triglyceride levels and cardiovascular diseases. First, we found that patients in the highest triglyceride quintile had a significantly greater risk of cardiac mortality than those in the lowest triglyceride quintile. Further, HR increased with the triglyceride quintile in a significant and dose-dependent manner, and high logarithm-transformed triglyceride levels were associated with increased long-term cardiac 
Table 1 Baseline characteristics

\begin{tabular}{|c|c|c|c|c|c|c|}
\hline & \multicolumn{5}{|c|}{ Triglyceride quintile, mmol/l } & \multirow[b]{2}{*}{$p$ Value $^{*}$} \\
\hline & $\begin{array}{l}\text { Q1 ( } \leq 1.11) \\
\mathrm{N}=369\end{array}$ & $\begin{array}{l}\text { Q2 (1.12-1.46) } \\
\mathrm{N}=359\end{array}$ & $\begin{array}{l}\text { Q3 (1.47-1.83) } \\
\mathrm{N}=369\end{array}$ & $\begin{array}{l}\text { Q4 (1.84-2.45) } \\
\mathrm{N}=370\end{array}$ & $\begin{array}{l}Q 5(\geq 2.46) \\
N=369\end{array}$ & \\
\hline Age, years & $60 \pm 9$ & $61 \pm 9$ & $59 \pm 8$ & $58 \pm 9$ & $58 \pm 8$ & $<0.001$ \\
\hline Men, n (\%) & $297(81)$ & $296(82)$ & $309(84)$ & $324(88)$ & $336(91)$ & $<0.001$ \\
\hline $\mathrm{BMI}, \mathrm{kg} / \mathrm{m}^{2}$ & $23 \pm 3$ & $23 \pm 3$ & $24 \pm 3$ & $24 \pm 3$ & $24 \pm 2$ & $<0.001$ \\
\hline Diabetes mellitus, n (\%) & $149(40)$ & $136(38)$ & $132(36)$ & 137 (37) & $149(40)$ & 0.913 \\
\hline Hypertension n (\%) & $243(66)$ & $238(66)$ & $245(66)$ & $257(70)$ & $255(69)$ & 0.211 \\
\hline Total cholesterol, mmol/l & $5.12 \pm 1.22$ & $5.53 \pm 1.11$ & $5.82 \pm 1.19$ & $5.92 \pm 1.34$ & $6.23 \pm 1.22$ & $<0.001$ \\
\hline HDL cholesterol, mmol/l & $1.24 \pm 0.36$ & $1.16 \pm 0.34$ & $1.11 \pm 0.31$ & $1.03 \pm 0.28$ & $0.98 \pm 0.28$ & $<0.001$ \\
\hline Non-HDL cholesterol, mmol/l & $3.88 \pm 1.16$ & $4.37 \pm 1.09$ & $4.71 \pm 1.13$ & $4.89 \pm 1.27$ & $5.25 \pm 1.20$ & $<0.001$ \\
\hline Current smoker, n (\%) & $260(71)$ & $243(68)$ & $272(74)$ & $280(76)$ & $304(82)$ & $<0.001$ \\
\hline Family history of CAD, $n(\%)$ & $126(34)$ & $119(33)$ & $102(28)$ & $103(28)$ & $125(34)$ & 0.446 \\
\hline Prior Ml, n (\%) & $147(40)$ & $175(49)$ & $170(46)$ & $185(50)$ & $204(55)$ & $<0.001$ \\
\hline Prior stroke, n (\%) & $15(4)$ & $24(7)$ & $11(3)$ & $14(4)$ & $18(5)$ & 0.720 \\
\hline Atrial fibrillation, $\mathrm{n}(\%)$ & $49(13)$ & $47(13)$ & $43(12)$ & $38(10)$ & $54(15)$ & 0.985 \\
\hline On dialysis (\%) & $6(1.6)$ & $1(0.2)$ & $6(1.6)$ & $7(1.9)$ & $7(1.9)$ & 0.283 \\
\hline No of diseased vessels & $2.17 \pm 0.84$ & $2.27 \pm 0.83$ & $2.24 \pm 0.85$ & $2.32 \pm 0.78$ & $2.28 \pm 0.82$ & 0.128 \\
\hline LMT lesion, n (\%) & $34(9)$ & $21(6)$ & $41(11)$ & $29(8)$ & $24(7)$ & 0.437 \\
\hline Arterial bypass to LAD, $n(\%)$ & $117(32)$ & $112(31)$ & $126(34)$ & $124(34)$ & $129(35)$ & 0.791 \\
\hline LVEF (\%) & $65.3 \pm 12.7$ & $64.0 \pm 12.9$ & $65.3 \pm 11.9$ & $63.9 \pm 13.5$ & $63.4 \pm 13.4$ & 0.136 \\
\hline Revascularisation-isolated $\mathrm{PCl}, \mathrm{n}(\%)$ & $139(38)$ & $117(33)$ & $96(26)$ & $98(27)$ & $85(23)$ & $<0.001$ \\
\hline \multicolumn{7}{|l|}{ Medications, n (\%) } \\
\hline Aspirin & $279(76)$ & $264(74)$ & $266(72)$ & $253(68)$ & $251(68)$ & 0.006 \\
\hline ACE inhibitors & $20(5)$ & $16(4)$ & $16(4)$ & $24(7)$ & $14(4)$ & 0.729 \\
\hline$\beta$-blockers & $88(24)$ & $84(23)$ & $113(31)$ & $123(33)$ & $111(30)$ & 0.003 \\
\hline Statins & $84(23)$ & $60(17)$ & $60(16)$ & $67(18)$ & $57(16)$ & 0.035 \\
\hline Fibrates & $3(0.8)$ & $8(2.2)$ & $10(2.7)$ & $5(1.4)$ & $7(1.9)$ & 0.551 \\
\hline Niacin & $37(10)$ & $29(8)$ & $28(8)$ & $37(10)$ & $23(6)$ & 0.214 \\
\hline
\end{tabular}

mortality even after adjustment for cholesterol levels and other covariates in secondary prevention of CAD. Second, the mortality risk of triglyceride was observed in patients with significant CAD who had achieved complete revascularisation. Finally, there were no interactions in each subgroup, although associations of fasting triglyceride level with cardiac mortality after complete revascularisation were obvious in men, patients with low HDL cholesterol levels and patients not receiving statins. Our findings therefore suggest that fasting triglyceride levels indicate mortality risk in the secondary prevention of CAD regardless of the presence or absence of other concomitant cardiovascular risks.
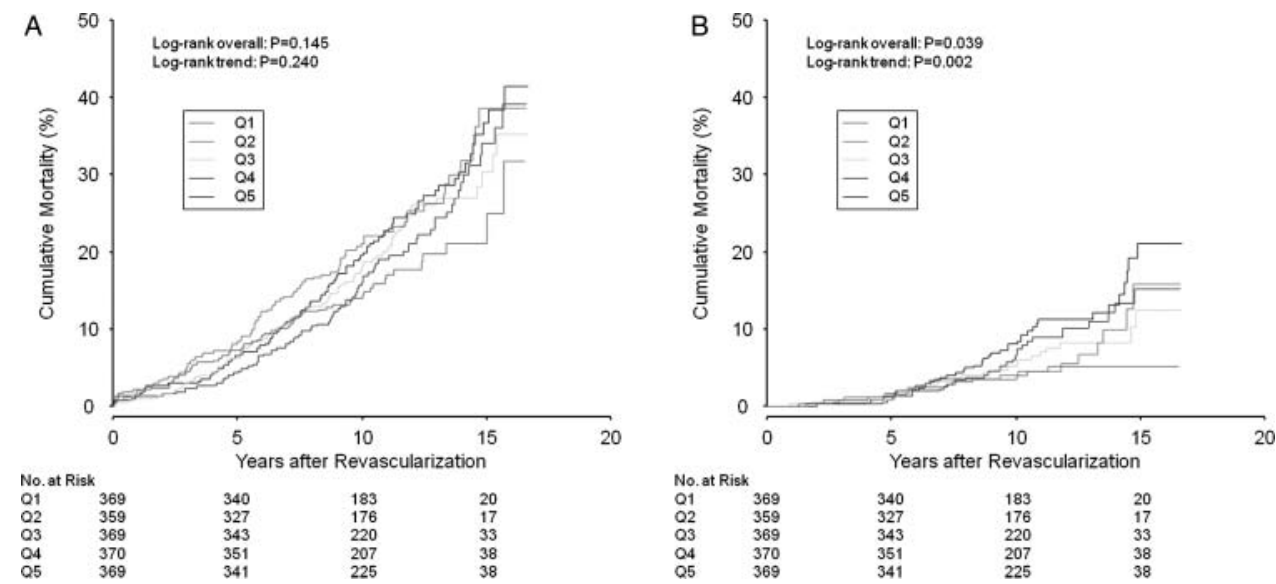

Figure 1 Cumulative mortality curves according to the quintiles of triglyceride levels for (A) all-cause deaths and (B) cardiac deaths. $p$ Values for overall log-rank tests indicate whether there is a difference in the five different mortality curves $(p=0.145$ for all-cause death, $p=0.039$ for cardiac death). $p$ Values for log-rank trend test indicate whether increased levels of triglycerides are associated with increased cumulative survival ( $p=0.240$ for all-cause death, $p=0.002$ for cardiac death). This figure is only reproduced in colour in the online version. 
A

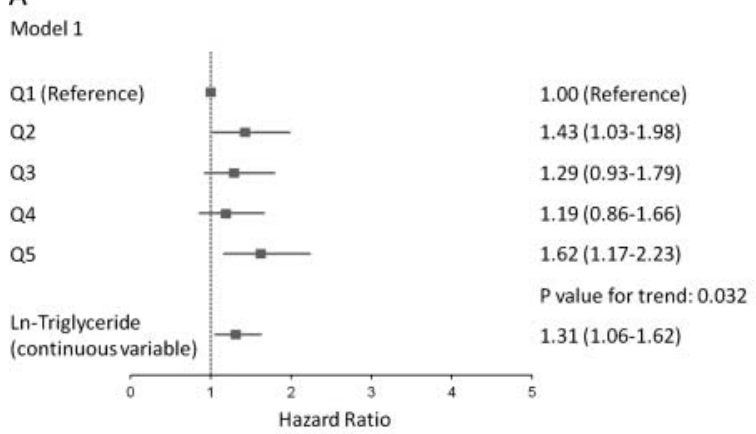

Model 2

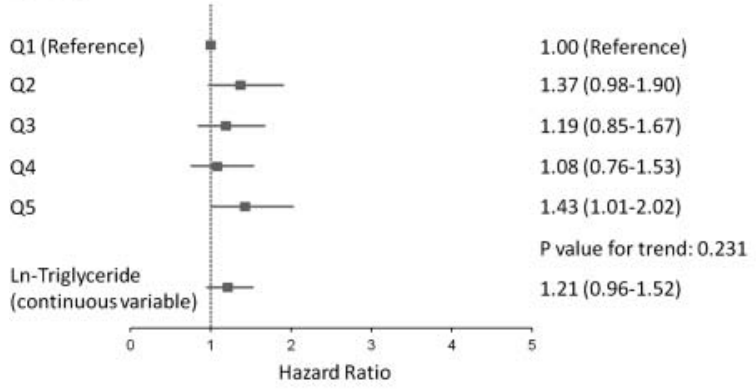

Model 3

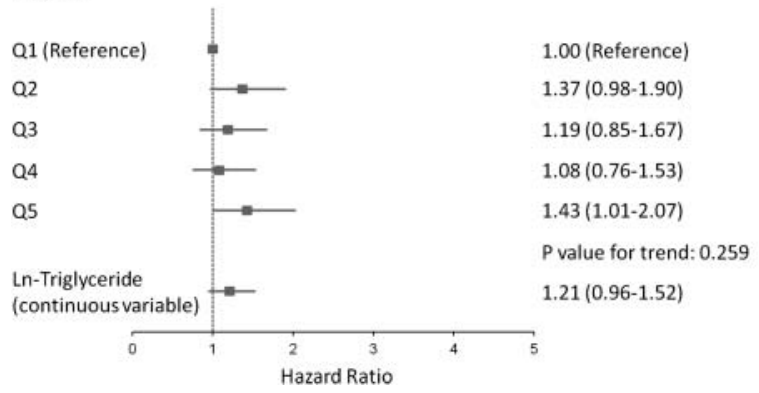

Model 4

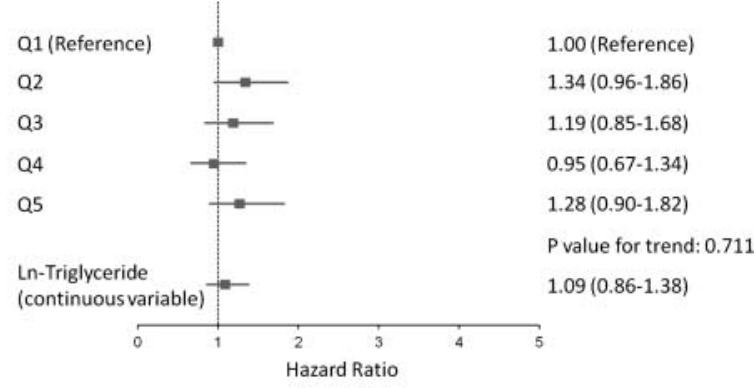

Model 5

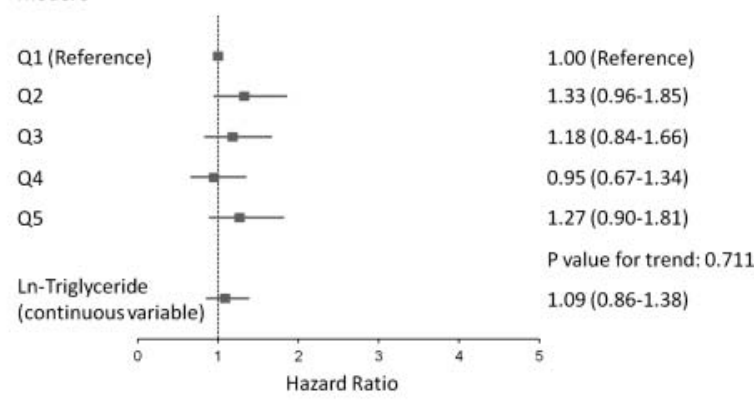

Figure 2 HR for mortality according to the quintiles of triglyceride levels: (A) all-cause deaths; (B) cardiac deaths. Model 1 adjusted for age and gender; Model 2 adjusted for age, gender, total and HDL cholesterols; Model 3 adjusted for age, gender, non-high-density lipoprotein (HDL) and HDL cholesterol; Model 4, adjusted for variables in model 2 plus hypertension, diabetes mellitus, prior myocardial infarction, prior stroke, atrial fibrillation, dialysis, left ventricular ejection fraction, number of diseased vessel, left main trunk lesion, isolated percutaneous coronary intervention, use of aspirin, use of angiotensin-converting enzyme (ACE) inhibitors, use of statins and use of niacin for all-cause death and the same variables other than hypertension, use of aspirin and use of ACE inhibitors for cardiac death; Model 5, adjusted for non-HDL cholesterol plus same variables in model 4 other than total cholesterol. Ln, natural logarithm-transformed. This figure is only reproduced in colour in the online version.

In the primary prevention of CAD the independent association of triglyceride levels with the morbidity and mortality rates of CAD has long been a controversial issue. ${ }^{11-13}$ In previous case-control studies, triglyceride levels have been identified as one of the risk factors for CAD even after adjustment for total and HDL cholesterol levels. ${ }^{14-17}$ Although most population-based cohort studies have shown a univariable association between triglyceride levels and $\mathrm{CAD}$, the relationship becomes non-significant or weak after adjustment for total and/or HDL cholesterol levels. ${ }^{13}$ There are at least four meta-analyses of population-based prospective studies regarding the relationships between triglyceride levels and morbidity and mortality rates of cardiovascular disease. ${ }^{5-8}$ Of these, three have similar conclusions. Hokanson and colleagues reported the results of a meta-analysis of 46413 men and 10864 women from the USA and European countries. ${ }^{5}$ In the univariable analysis the relative risk of triglyceride (per $1 \mathrm{mmol} / \mathrm{l}$ ) for the composite of fatal and non-fatal cardiovascular disease was 1.32 in men and 1.76 in women. After adjustment for HDL cholesterol, these relative risks were attenuated to the modest levels of 1.14 in men and 1.37 in women. A recent updated meta-analysis that examined 262525 subjects from the USA and European countries revealed a 1.7 times higher risk for the composite of fatal and non-fatal CAD at the upper triglyceride tertile compared with the lower triglyceride tertile in the adjusted analysis. ${ }^{7}$ Another meta-analysis that examined 96224 men and women from the Asian and Pacific populations showed that the risk for the composite of fatal and non-fatal CAD in individuals in the top triglyceride quintile was 1.8 times greater than those in the bottom triglyceride quintile after adjustment for several established risk factors. ${ }^{6}$ In the most recent and robust evidence from the Emerging Risk Factors Collaboration, 302430 people were examined in 68 prospective studies. ${ }^{8}$ With adjustment for age and sex, triglycerides showed a strong stepwise association with fatal and non-fatal CAD. However, after adjustment for standard risk factors and other lipid measures such as non-HDL and HDL cholesterol levels, the association between triglycerides and CAD was no longer significant. ${ }^{8}$ The American Heart Association has recently suggested that the independence of the triglyceride level as a causal factor in developing CAD remains debatable, but triglyceride levels appear to provide unique information and can be used as a biomarker of risk. ${ }^{18}$ 

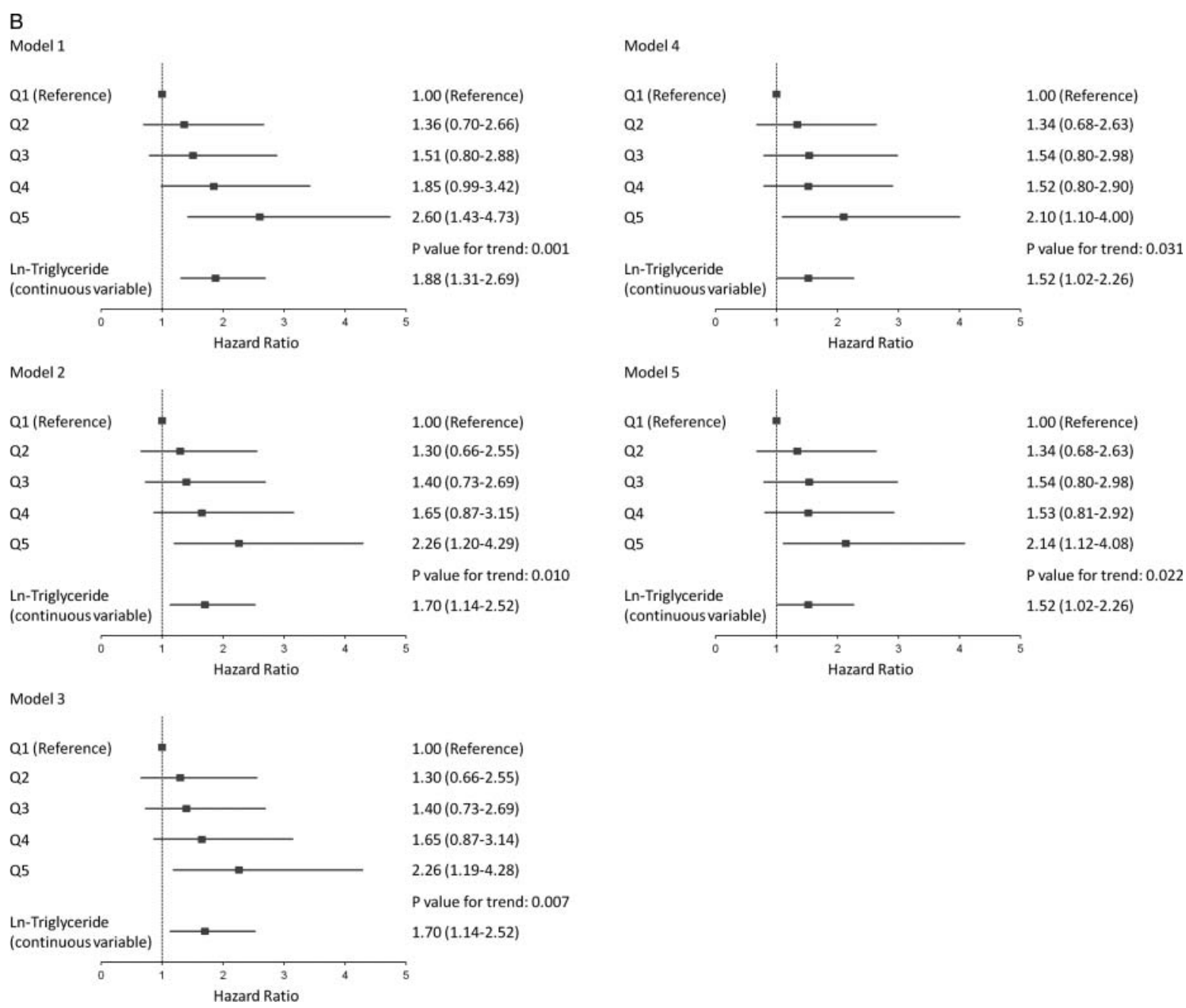

Figure 2 Continued.

Although individuals enrolled in some of the populationbased prospective studies included in the abovementioned meta-analysis had a history of CAD, the percentage of such individuals was very low $(<15 \%)$. Furthermore, there are no subdivided analyses regarding individuals with CAD. In the case of secondary prevention of CAD, only one report suggesting a significant relationship between triglyceride levels and long-term prognosis is available. von Eynatten and colleagues reported that, in patients with CAD, fasting triglyceride levels were associated with a high incidence of secondary cardiovascular events (ie, composite of cardiovascular death, non-fatal MI and stroke) during a median follow-up term of 57 months, even after adjustment for other lipid and adiponectin levels, with an HR of 1.5 which is identical to the results of our study (as a continuous variable). ${ }^{19}$ However, the main purpose of their study was not to assess the relationship between triglyceride levels and prognosis but to investigate whether adiponectin is a useful prognostic predictor in patients with $\mathrm{CAD}$ and to compare the values of adiponectin for secondary risk stratification with the prognostic role of markers of dyslipidaemia (ie, triglyceride, low-density lipoprotein (LDL) and HDL cholesterol). Their patients were a mixture of those who had undergone non-invasive or invasive (PCI and CABG) treatment. Except for the severity of CAD, the details related to CAD and type of treatment were not specifically described and were not adjusted for in the multivariable analysis (eg, whether PCI was successful or whether complete revascularisation was performed were not mentioned and no adjustment was made for them). Our study shows that, in patients with complete revascularisation, fasting triglyceride levels were associated with increased cardiac mortality for a long-term follow-up period ( $>10$ years). It was important to assess data only from patients who had achieved complete revascularisation because initial CAD events may be prevented or delayed by complete coronary revascularisation, even in patients with severe coronary atherosclerosis. This selection minimises the bias of treatment procedures for initial CAD events. Therefore, we specifically assessed the effect of fasting triglyceride levels on long-term mortality among the secondary prevention cohort of patients with CAD in this study.

We also assessed the possible interactions of triglyceride levels and cardiac mortality with age, gender, presence or absence of DM, total and HDL cholesterol and use of statins. There were no statistically significant interactions between the subgroups, which indicated that the relationship between triglyceride levels and high cardiac mortality was not affected by these factors. A strong relationship was found between triglyceride levels and cardiac mortality in men, patients with low HDL and patients not receiving statins. Although the Framingham Heart Study suggested that a high triglyceride level was a predictor of the incidence of cardiovascular disease in women, ${ }^{20}$ the triglyceride level in our study did not show a significant relationship with increased cardiac mortality in women with CAD. In general, 
A
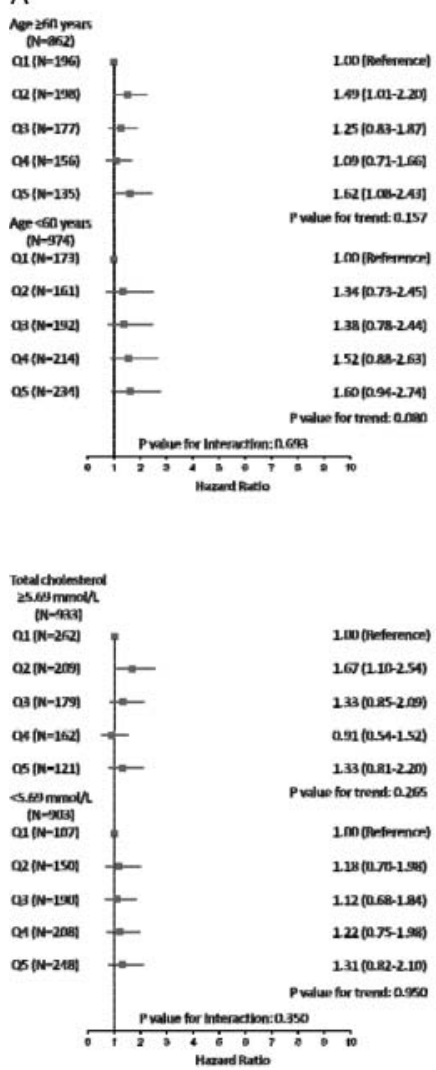
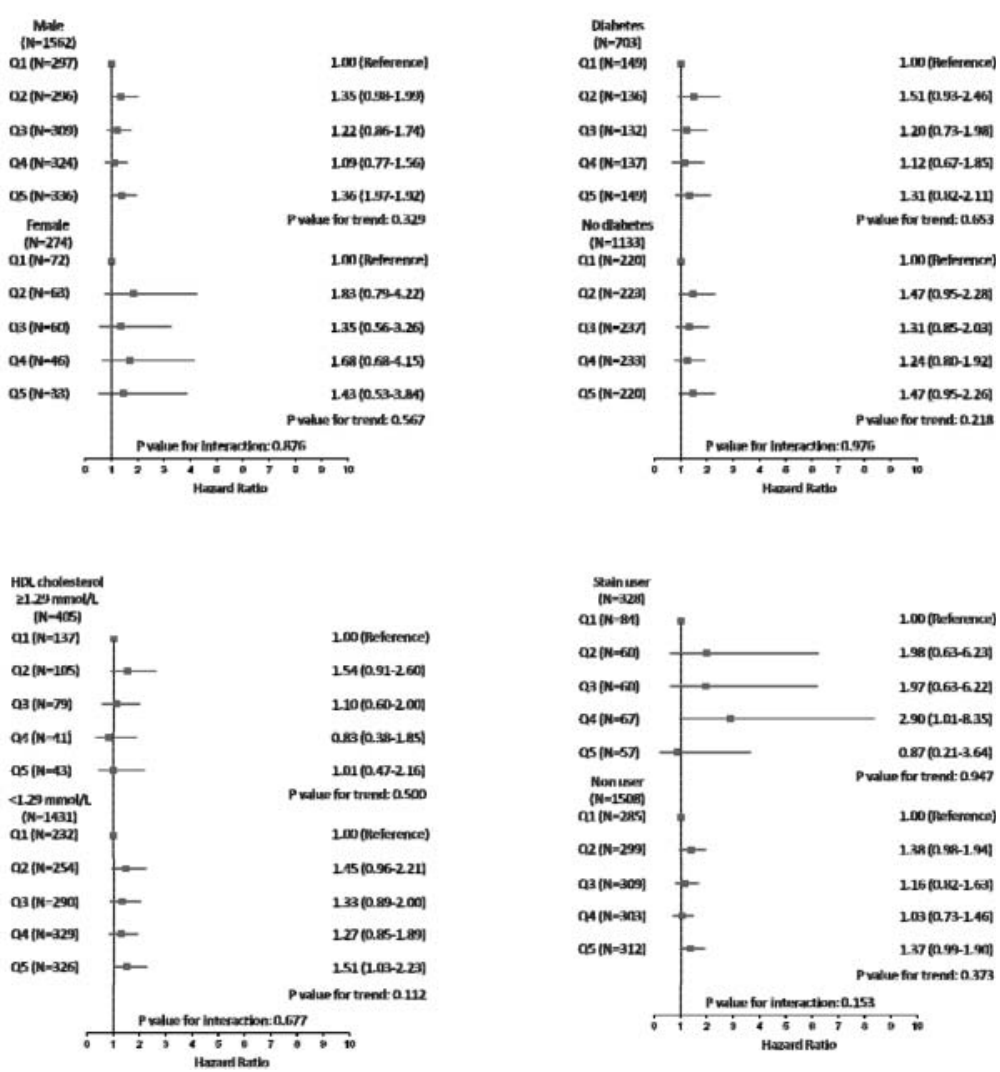

Figure 3 Subgroup analyses of (A) all-cause deaths and (B) cardiac deaths. HDL, high-density lipoprotein. This figure is only reproduced in colour in the online version.

women who receive coronary revascularisation are older and their baseline risk profiles are worse than men. ${ }^{21} 22$ These factors might attenuate the significance of the association of triglyceride levels and cardiac mortality in women in our study population. Nevertheless, considering that no significant association was observed between triglyceride levels and cardiac mortality in all subgroups which included small numbers of patients (ie, women, patients with high HDL cholesterol levels and patients receiving statins), the results of analysis within individual subgroups should be interpreted with caution.

On the other hand, the relationship between triglyceride levels and mortality risks among patients with CAD receiving statin treatment has been investigated in several studies. For instance, Wolfram et $a l^{23}$ reported that, in patients with acute coronary syndrome of whom $98 \%$ were on statin treatment, triglyceride levels were not associated with 1-year clinical outcomes. In addition, there was no significant relationship between triglyceride levels and short-term outcome in the Myocardial Ischaemia Reduction with Aggressive Cholesterol Lowering trial. ${ }^{24}$ Analyses from the Incremental Decrease in End Point Through Aggressive Lipid Lowering trial and the Treating to New Targets trial showed that triglyceride levels are associated with a risk of cardiovascular events even after adjustment of other lipid parameters, but this relationship was no longer significant when other risk factors were included in further multivariable analysis. ${ }^{25}$ In contrast, the Pravastatin or Atorvastatin Evaluation and Infection Therapy-Thrombolysis In Myocardial Infarction (PROVE IT-TIMI) 22 trial showed an independent relationship between triglyceride levels and cardiovascular events outcome, which is consistent with the results of the present study. ${ }^{26}$ These conflicting results could be explained by the differences in baseline triglyceride levels across these studies, including ours. In studies which failed to show a significant relationship between triglyceride levels and outcomes, ${ }^{23-25}$ patients had relatively low triglyceride levels compared with the PROVE IT-TIMI 22 trial $^{26}$ and the present study, suggesting that the degree of hypertriglyceridaemia may affect the effect of adjustment for covariates including other lipid parameters. Nevertheless, these results also indicate that elevated triglyceride levels can be a predictor of worse outcomes even in patients on statin treatment, and further adjunctive intervention for elevated triglyceride levels should be considered. ${ }^{27}$

It remains controversial whether there is a causal relationship between triglyceride levels and CAD morbidity and mortality. The triglyceride level is rather regarded as an important biomarker of cardiovascular disease because of its association with atherogenic remnant particles and apo CIII. ${ }^{18}$ Randomised controlled studies on treatment for lowering triglyceride levels could provide a solution to this controversy. However, all available interventions for lowering triglyceride levels such as drugs (eg, fibrates, niacin and statins) and lifestyle modifications also affect the confounding parameters, including LDL cholesterol, HDL cholesterol and insulin resistance, ${ }^{28-31}$ so we could not determine the causality in such studies. However, as the condition is characterised by an increased circulating triglyceride level, the triglyceride level can be considered as an interventional target. This hypothesis is supported by a recent report by Tirosh and colleagues ${ }^{32}$ who followed 13953 young men aged 26-45 years for 5.5 years and performed two measurements of fasting triglycerides 5 years apart. There were significant correlations between a good lifestyle and the reduction in triglyceride levels between the two measurements. Evaluation of the change 

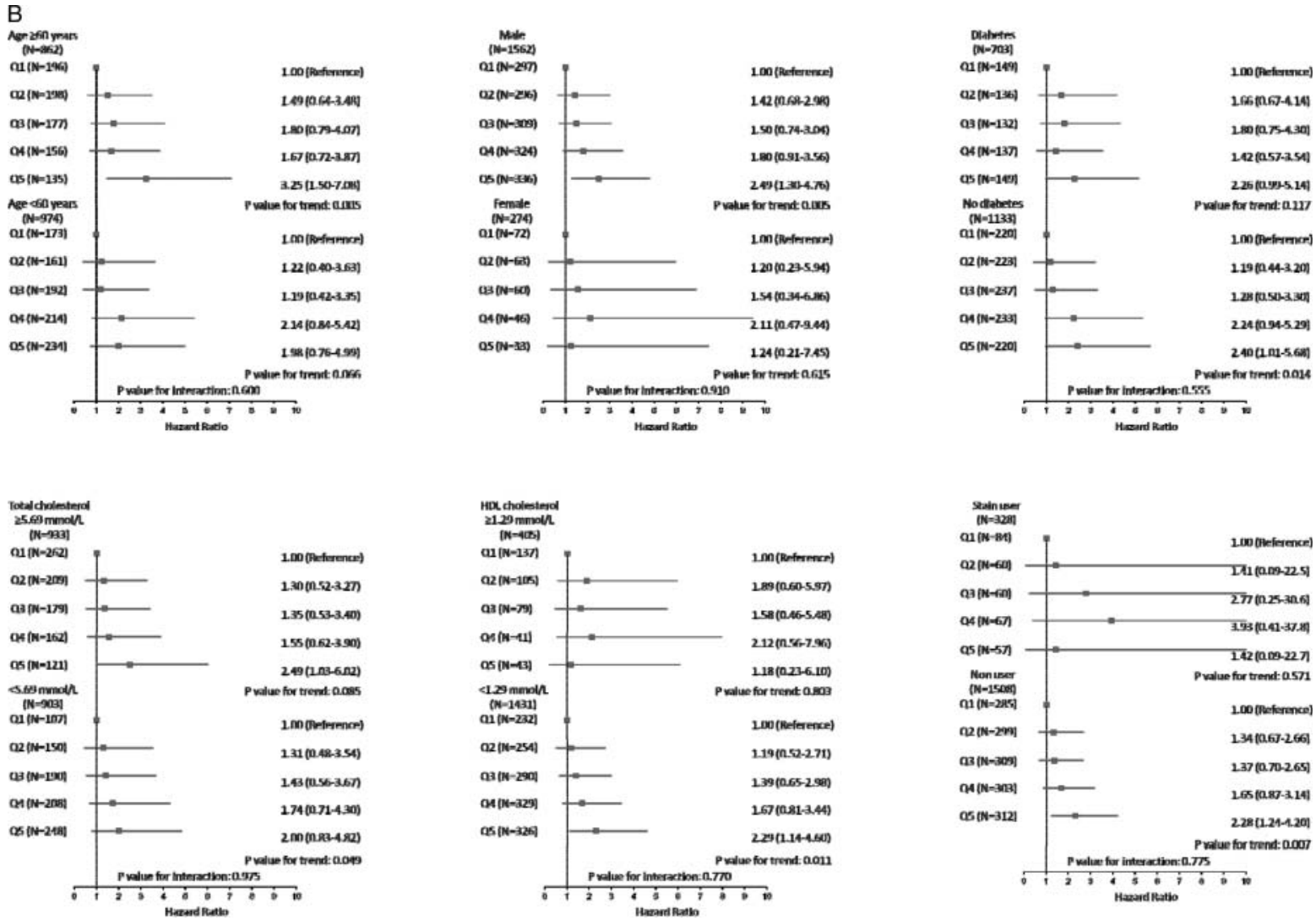

Figure 3 Continued.

in triglyceride levels over the first 5 years and incident CAD in the next 5.5 years showed a direct correlation between increases in triglyceride levels and the risk of CAD. These findings suggest a causal relationship between increased triglyceride level and CAD morbidity and mortality.

The present study has several limitations. First, balloon angioplasty was the only PCI used in all patients and $51.1 \%$ of the CABG procedures involved an arterial graft. It is difficult to determine whether the use of stents and arterial grafts could have improved the results in the recent era of revascularisation and to evaluate the relative importance of improvements in both operator skills and adjunctive drug therapy. Further investigation is needed to clarify whether triglyceride levels will affect the long-term mortality in the stent and arterial bypass era. Second, assessment of data only of patients who achieved complete revascularisation also introduced potential selection bias in terms of the overall mortality rate which should be taken into account. Third, several groups recently reported that the nonfasting triglyceride level is a superior predictor of cardiovascular risk than fasting levels. ${ }^{33}{ }^{34}$ If we can use non-fasting triglyceride levels as a predictor of cardiovascular morbidity and mortality in patients with CAD, it would have greater clinical applications as it is easier to obtain non-fasting than fasting triglyceride levels. Further studies and discussions regarding the importance of non-fasting triglyceride levels in the secondary prevention of CAD are therefore needed.

\section{Conclusions}

Fasting triglyceride levels were associated with an increase in cardiac mortality over a 10 -year period after complete coronary revascularisation. This association was observed even after adjustment for the total and HDL cholesterol levels together with other covariates.

Contributors HD had full access to all data in the study and takes responsibility for the integrity of the data and the accuracy of the data analysis. Conception and design: HD, TK, KM. Acquisition of data: KK, NK, MO, ST. Analysis and interpretation of data: HD, TK, NY. Drafting of the manuscript: TK, NY. Critical revision of the manuscript for important intellectual content: $H D, K M, A A$. Final approval of the version to be published: HD, KM, KK, NK, MO, ST, AA.

Competing interests None.

Ethics approval Ethics approval was obtained from Juntendo University ethics committee.

Provenance and peer review Not commissioned; externally peer reviewed.

\section{REFERENCES}

1 Assmann $\mathrm{G}$, Schulte $\mathrm{H}$, Funke $\mathrm{H}$, et al. The emergence of triglycerides as a significant independent risk factor in coronary artery disease. Eur Heart J 1998;19 (Suppl M):M8-14.

2 Jeppesen J, Hein HO, Suadicani P, et al. Triglyceride concentration and ischemic heart disease: an eight-year follow-up in the Copenhagen Male Study. Circulation 1998:97:1029-36.

3 Stavenow L, Kjellstrom T. Influence of serum triglyceride levels on the risk for myocardial infarction in 12,510 middle aged males: interaction with serum cholesterol. Atherosclerosis 1999;147:243-7.

4 Criqui $\mathrm{MH}$, Heiss $\mathrm{G}$, Cohn R, et al. Plasma triglyceride level and mortality from coronary heart disease. N Engl J Med 1993;328:1220-5.

5 Hokanson JE, Austin MA. Plasma triglyceride level is a risk factor for cardiovascular disease independent of high-density lipoprotein cholesterol level: a meta-analysis of population-based prospective studies. J Cardiovasc Risk 1996;3:213-19.

6 Patel A, Barzi F, Jamrozik K, et al. Serum triglycerides as a risk factor for cardiovascular diseases in the Asia-Pacific region. Circulation 2004;110:2678-86.

7 Sarwar N, Danesh J, Eiriksdottir G, et al. Triglycerides and the risk of coronary heart disease: 10158 incident cases among 262525 participants in 29 Western prospective studies. Circulation 2007;115:450-8.

8 Di Angelantonio E, Sarwar N, Perry P, et al. Major lipids, apolipoproteins, and risk of vascular disease. JAMA 2009;302:1993-2000. 
9 Jones EL, Weintraub WS. The importance of completeness of revascularization during long-term follow-up after coronary artery operations. J Thorac Cardiovasc Surg 1996;112:227-37.

10 McLellan CS, Ghali WA, Labinaz M, et al. Association between completeness of percutaneous coronary revascularization and postprocedure outcomes. Am Heart J 2005; 150:800-6

11 Brown DF, Kinch SH, Doyle JT. Serum triglycerides in health and in ischemic heart disease. N Engl J Med 1965;273:947-52.

12 Hulley SB, Rosenman RH, Bawol RD, et al. Epidemiology as a guide to clinical decisions. The association between triglyceride and coronary heart disease. $N$ Engl J Med 1980;302:1383-9.

$13 \mathrm{NIH}$ Consensus conference. Triglyceride, high-density lipoprotein, and coronary heart disease. NIH consensus development panel on triglyceride, high-density lipoprotein, and coronary heart disease. JAMA 1993;269:505-10.

14 Castelli WP, Doyle JT, Gordon T, et al. HDL cholesterol and other lipids in coronary heart disease. The cooperative lipoprotein phenotyping study. Circulation 1977;55:767-72

15 Fager G, Wiklund O, Olofsson SO, et al. Multivariate analyses of serum apolipoproteins and risk factors in relation to acute myocardial infarction. Arteriosclerosis 1981;1:273-9.

16 Kukita H, Imamura Y, Hamada M, et al. Plasma lipids and lipoproteins in Japanese male patients with coronary artery disease and in their relatives. Atherosclerosis 1982;42:21-9.

17 Reardon MF, Nestel PJ, Craig IH, et al. Lipoprotein predictors of the severity of coronary artery disease in men and women. Circulation 1985;71:881-8.

18 Miller M, Stone NJ, Ballantyne C, et al. Triglycerides and cardiovascular disease: a scientific statement from the American Heart Association. Circulation 2011;123:2292-333.

19 von Eynatten M, Hamann A, Twardella D, et al. Atherogenic dyslipidaemia but not total- and high-molecular weight adiponectin are associated with the prognostic outcome in patients with coronary heart disease. Eur Heart J 2008;29:1307-15.

20 Castelli WP. The triglyceride issue: a view from Framingham. Am Heart 1986;112:432-7.

21 Fisher LD, Kennedy JW, Davis KB, et al. Association of sex, physical size, and operative mortality after coronary artery bypass in the Coronary Artery Surgery Study (CASS). J Thorac Cardiovasc Surg 1982;84:334-41.
22 Mehilli J, Kastrati A, Dirschinger J, et al. Differences in prognostic factors and outcomes between women and men undergoing coronary artery stenting. JAMA 2000:284:1799-805.

23 Wolfram RM, Brewer HB, Xue Z, et al. Impact of low high-density lipoproteins on in-hospital events and one-year clinical outcomes in patients with non-ST-elevation myocardial infarction acute coronary syndrome treated with drug-eluting stent implantation. Am J Cardiol 2006;98:711-17.

24 Olsson AG, Schwartz GG, Szarek M, et al. High-density lipoprotein, but not low-density lipoprotein cholesterol levels influence short-term prognosis after acute coronary syndrome: results from the MIRACL trial. Eur Heart $J$ 2005;26:890-6.

25 Faergeman 0, Holme I, Fayyad R, et al. Plasma triglycerides and cardiovascular events in the Treating to New Targets and Incremental Decrease in End-Points Through Aggressive Lipid Lowering trials of statins in patients with coronary artery disease. Am J Cardiol 2009;104:459-63.

26 Miller M, Cannon CP, Murphy SA, et al. Impact of triglyceride levels beyond low-density lipoprotein cholesterol after acute coronary syndrome in the PROVE IT-TIMI 22 trial. J Am Coll Cardiol 2008:51:724-30.

27 Watts GF, Karpe F. Triglycerides and atherogenic dyslipidaemia: extending treatment beyond statins in the high-risk cardiovascular patient. Heart 2011;97:350-6.

28 Preiss D, Sattar N. Lipids, lipid modifying agents and cardiovascular risk: a review of the evidence. Clin Endocrinol (Oxf) 2009;70:815-28.

29 Pasanisi F, Contaldo F, de Simone G, et al. Benefits of sustained moderate weight loss in obesity. Nutr Metab Cardiovasc Dis 2001;11:401-6.

30 Durstine JL, Grandjean PW, Cox CA, et al. Lipids, lipoproteins, and exercise. J Cardiopulm Rehabil 2002;22:385-98.

31 Fontana L, Villareal DT, Weiss EP, et al. Calorie restriction or exercise: effects on coronary heart disease risk factors. A randomized, controlled trial. Am J Physiol Endocrinol Metab 2007;293:E197-202.

32 Tirosh A, Rudich A, Shochat $T$, et al. Changes in triglyceride levels and risk for coronary heart disease in young men. Ann Intern Med 2007;147:377-85.

33 Nordestgaard BG, Benn M, Schnohr P, et al. Nonfasting triglycerides and risk of myocardial infarction, ischemic heart disease, and death in men and women. JAMA 2007;298:299-308.

34 Bansal S, Buring JE, Rifai N, et al. Fasting compared with nonfasting triglycerides and risk of cardiovascular events in women. JAMA 2007:298:309-16. 